WORK IN PROGRESS

\title{
Exergames to study psychomotor factors of children with ADHD: Preliminary findings
}

\author{
Marisela Hernández, Estefany Terrazas, Fernando Martínez, Raymundo \\ Cornejo, Vania Álvarez, Claudia Barraza, Fernando Gaxiola
}

Published: 30 November 2020

\begin{abstract}
Exergaming is considered an effective way to promote physical exercising for young children. Previous work has argued in favor of using exergaming to help with health problems such as obesity, or to contribute with brain maturation, which may affect motor and cognitive abilities. In this work, we describe the preliminary results of a study focused on observing the psychomotor factors exposed by a child with ADHD attending a public primary school. The case of study is an eleven-year-old girl who attends a public primary school but who receives academic support from special education therapists. An off-the-shelf exergame was selected based on its instructional and physical activation features, which the child played with for six weeks. The exergame meets its aim to stimulate psychomotor factors. Attention, speed of reaction, body posture, temporospatial and dissociation skills are categories used for video analysis. Preliminary results seem to indicate that the child's interaction with the game has produced positive outcomes for reading comprehension, writing, memory, and visuomotor skills. CUMANES and MABC-2 are the batteries used for PRE and POST tests.
\end{abstract}

\section{Keywords}

Learning disabilities; Exergames; Psychomotor Skills.

\section{Introduction}

There are studies indicating that physical activity and academic performance can be correlated. Problems with gross and fine motor skills could lead to problems with literacy and mathematics [20], poor locomotor skills affect reading and poor object control affects math's [25]. Poor skills with dynamic/static balance, reaction speed and power are also associated to low academic performance [18]. Given the relationship between academic performance and physical activation there is a great concern about the opportunities of students to do physical activity inside and outside the school [9], [26].

\footnotetext{
Hernández, M., Terrazas, E.

Ciencias de la Cultura Física

Universidad Autónoma de Chihuahua

a292744@uach.mx, a308769@uach.mx
}

Martínez, F., Cornejo, R., Álvarez, V., Barraza, C., Gaxiola, F. Facultad de Ingeniería

Universidad Autónoma de Chihuahua

fmartine@uach.mx,rcornejo@uach.mx, valvarez@uach.mx,

cbarraza@uach.mx,lgaxiola@uach.mx
Exergaming is a term used for video games which allow for doing a form of exercise, helping to promote an active lifestyle. Exergames can raise the heart rate and help with energy expenditure, which might produce improvements with mental health and reduce obesity [12]. Physical activation using exergames might help children with developmental or learning disabilities. The exergames can be used to make the children aware of the benefits of exercising [10], to help them practicing cognitive functions [7], or even to improve behavior [6].

Most of the exergames used to support disabled people are ad hoc designs and focused on attending specific children challenges. However, because the exergame market is increasing its availability for families, it is important to understand whether children with, for instance, learning disabilities, may get similar benefits to those of neurotypical children. That is, whether exergaming could help children exercise and workout psychomotor components (e.g. temporospatial awareness and quality of movements), and by means of these enhance their learning skills.

\section{Related work}

Exergames or games that combine play and exercise have a long history and its market rises constantly. In the 70's and 80's video arcades games such as Pac Man and Pole Position, demanded that the player stood up the full gameplay, and the players themselves made great body gestures and articulations to have a vivid gameplay experience [3]. The NES game (Nintendo Entertainment System), by the 90's, was one of the first games that allowed for hand or foot interaction. Athletic World, for example, requires the player to run short distances, to stand still or to shift to different positions on a floor pad, representing orthogonal physical action. Physical-active gameplay was reproduced in games such as Yourself! Fitness to motivate physical exertion.

\subsection{Exergames and Physical Activation}

Nowadays exergames are considered as a resource to support the development of motor skills, the promotion of a healthy lifestyle or the learning of sport skills [11]. As cited previously, this genre of game besides its contribution to physical activation can help to enhance children behavioral components, such as tolerance to challenges, which could facilitate people be more sociable.

In schools, exergaming is seen as a motivator to engage children in the physical-education class [23]. In a study, researchers augmented sport equipment and video games consoles to let children play active games. By using their arms, legs, or wholebody movements children played tennis, boxing, football, baseball, biking, among others. Their physical activity level, caloric expenditure, and engagement with physical education activities 
were measured. Reported results state that exergames can offer moderated physical activity, and that exergame-based physical activation was preferred instead of cultural configurations of physical education classes. Sheehan and Katz [22] used an exergame to motivate children enrolled in the fourth year of primary school, to do exercise for 24 minutes, 5 days a week for 6 weeks, aiming to enhance body posture.

\subsection{Exergames and Cognition}

Developmental researchers are interested in cognitive processes, and the optimal use of executive functions (EF) to solve the task at hand [2]. EF is needed, for example, to self-direct learning and selfregulate emotions. A disorder on EF can make problematic the children transition to formal schooling. In his work, the author compared results from children that played physical-active exergames versus sedentary video games and found that even short periods of intense activity with exergaming enhances EF. Karime et al. [16], for instance, build a system named Learn-Pads that consists of pressure sensors, which children need to step on to solve some basic arithmetic operations such as addition and multiplication. Tomporowski et al. [24] suggest that when physical activation requires that children use multi-limb movements and rapid decision-making, it is possible to assume tight mental engagement. According to these authors, children that practice the bouncing of a volleyball alternating hands at different speed, can perform better than those who participated in a normal class of physical education without specific body's coordination demands.

\subsection{Exergames and Children with Special Needs}

Exergaming has been seen also a tool to stimulate visual, auditive and kinesiology channels, and to improve learning skills of children with gross motor skills impairments [1]. Children with dyspraxia and other related disorders such as autism, Asperger's Syndrome and Attention Deficit Disorder, can use hands or any other body's limbs to interact with exergames in order to enhance their skills for attention, coordination, spatiality and event reaction. Bossavit and Pina [5] configured activities for painting, object discovery and music creation to improve stabilization and coordination of children with motor and cognitive disabilities. The authors pointed out that children with disabilities cannot compete with neurotypical peers, so the built system considered each individual profile to adapt the gameplay and offer the best exergaming experience for this group of children.

Hwang et al. [15] work suggests that the video game market may be excluding children with special needs. First, because the augmented devices that enable interaction with video games cannot be used for children with, for instance, cerebral palsy. In their study, a special bike was built to allow children use pedals to interact with a race game that demands gross motor skills efforts, and with a shooting game that demands aiming and fine motor skills. Second, because people with cerebral palsy present different levels of physical abilities, the gameplay was dynamically adjusted to take into account players' speed of interaction response.

\section{Methodology}

The aim of this paper is to explore the extent to which psychomotor factors such as spatiotemporal orientation and quality of movement can be stimulated using off-the-shelf exergames, given the literature evidence that executive functions are positively affected. Then, the first approach was to select an exergame based on its instructional characteristics and the level of psychomotor factors demanded for the game logic.

\subsection{Exergame Selection}

First, we selected the adventures over the dance and sports Xbox games considering the human body parts needed to interact with the game, and the level of interactions the game would demand from children. Second, besides its interaction features, the adventures game includes characters, challenges, clear rules to complete activities and to some extent, let the user have control of the game; all, these in addition to the provide sensory stimulation [13], as observed in Table 1.

Table 1. Games selected and their instructional features

\begin{tabular}{|l|c|c|c|c|}
\hline Features & $\begin{array}{c}\text { Reflex } \\
\text { Ridge }\end{array}$ & $\begin{array}{c}20,000 \\
\text { Leaks }\end{array}$ & River Rush & Rally Ball \\
\hline Fantasy & $*$ & $*$ & $*$ & $*$ \\
\hline Rules & $*$ & $*$ & & \\
\hline $\begin{array}{l}\text { Sensory } \\
\text { Stimulation }\end{array}$ & $*$ & $*$ & $*$ & $*$ \\
\hline Challenges & $*$ & $*$ & $*$ & \\
\hline Mystery & $*$ & $*$ & $*$ & $*$ \\
\hline Control & $*$ & $*$ & $*$ & \\
\hline
\end{tabular}

Reflex Ridge make the player feel the sensation of a fantasy race. The level of immersion would arise sensations of being playing at a thematic park. There are clear rules of how the players can get coins and the way they can complete the game. Visual and auditory stimuli help the player make decisions along the game play. The collection of coins is not often an easy task due to the obstacles the player should avoid during the race. Nevertheless, the player can discover how to control her body in order to response to the game stimulus, get the better experience and most of the points in the game.

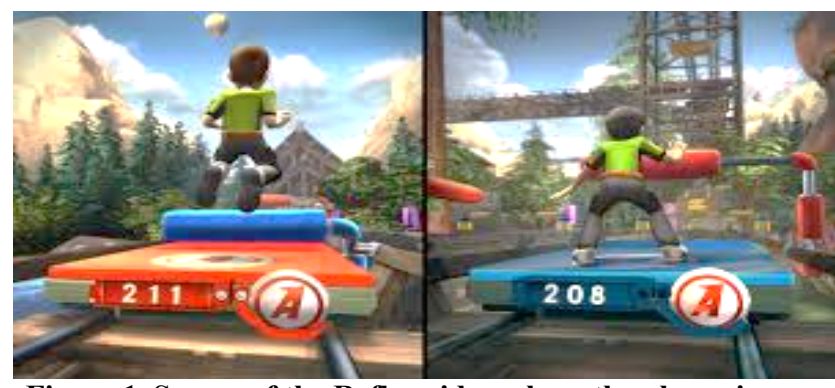

Figure 1. Scenes of the Reflex ridge where the player jumps (left) or moves to the left side (right) to avoid obstacles.

Figure 1, shows some of the psychomotor factors the player needs to use to, for example, calculate the distance and the time to jump over the bar, or to use spatial awareness to avoid objects coming to the right or left side of the player's avatar.

Players understand that much of the success for this game would depend on the way they use their body, and psychomotor factors, to attend the game logic. As shown by Figure 2, the player needs also to extend arms, or to coordinate equilibrium with arms organization to reach the coins. 


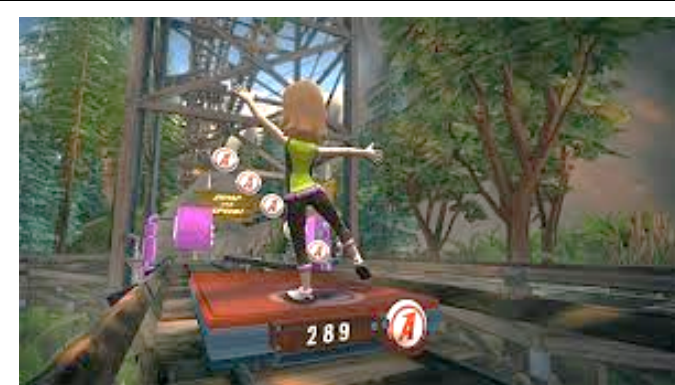

Figure 2. Game logic demanding equilibrium and body dissociation capabilities.

This genre of exergames, therefore, demands different levels of interactions that would help children practice motor coordination, equilibrium, laterality, working memory, visuomotor perception, and speed of reaction.

\subsection{Case of Study Participants Selection}

The subject is an eleven-year-old girl with diagnosis of ADHD since year two after born. She is enrolled at the fifth year of a public primary school and receives academic support from USAER (Service Unit that Support Regular Education), a Mexican institution that support children with special education needs. This government department helps children to develop academic competences. Children can receive technical, methodological, and psychological support within the school premises and within the normal school hours. Services provided by USAER can be extended to parents and teachers. The child receives support with reading comprehension, individual and social-affective behavior, and gross motor limitations.

\subsection{Baseline}

In order to get information about her cognitive and psychomotor challenges two standardized tests were applied: CUMANES and MABC-2. CUMANES is a test that assesses comprehension and reading speed. A small text is handed out to a child for reading, when the task is completed the time used for it is registered and several questions related to, for instance, passages and characters are asked. In another subtest the child is asked to copy figures on a notebook and the preferred side of his/her body used to complete the activity is observed to identify laterality. MABC-2 is a test that evaluates manual dexterity. It consists of exercises the child must reproduce using her hands, fingers, and visual coordination to, for instance, catching a ball. Observations from motor skills activities are classified as clumsiness, slowness, and inaccuracy.

\subsection{Exergaming}

A classroom of the School of the Science for the Physical Culture was used to integrate the technology and to offer the child the experience with exergames, see Figure 3.

The Kinect device and an Xbox 360 console were used to play the exergames. A Kinect device version two, connected to a computer, was used to collect raw data from the player skeleton. A projector displayed the exergame on the wall. A polar heart rate band wirelessly connected to a Texas Instrument ez-Chronos watch was used to monitor cardiac frequency, as an indirect measure of breathing, and a physiological measure associated to muscular tone issues [4]. Two physical education therapists directed each of the exergaming sessions, and one of them used a tablet to tag the body limbs used by the player while exergaming. In addition, video footage was recorded for the posterior analysis of psychomotor factors that are not evaluated with the standard tests.

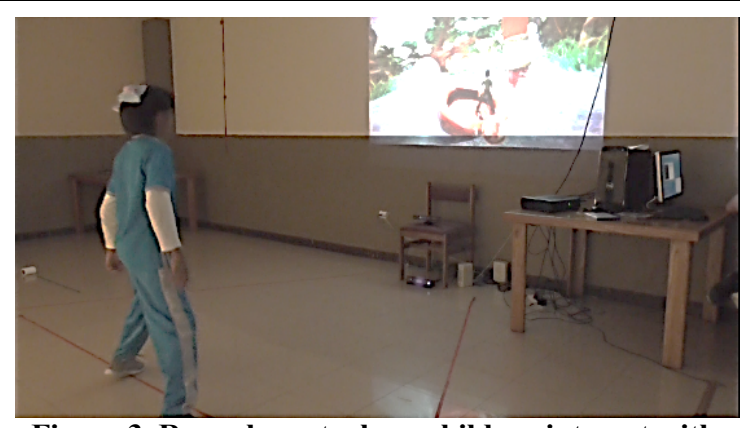

Figure 3. Room layout where children interact with exergames.

\section{Results}

As established previously, the aim of the study was to observe psychomotor components such as spatiotemporal orientation and quality of movement, which are not often evaluated on exergaming. Spatiotemporal orientation in combination with movement, balance and motor reaction form the coordinative skills [19], and quality of movement, QoM, is considered as the body ability to reproduce movements related to time and space [8]. One important QoM component is the muscle tone, the continuous and partial contraction of the muscles, a physiological factor that the human being uses and orient to complete motor related activities [17], including academic ones such as writing and mathematics. Furthermore, poor muscle tone or bad articulations of muscles can yield bad postures, which contributes to problems with balance and functional mobility [21].

We studied the interaction with exergames of a child with learning disabilities in order to seek for the level of stimulation this genre of games offers for psychomotor factors, and the extent to which learning abilities can be enhanced. To study psychomotor factors a video analysis of about twenty sessions was done. In this section, we share findings that inform about the evolution of the child based on her interaction with the reflex ridge game considering three moments of the intervention phase. For each moment, the game difficulty varies, at least regarding the game time and the interaction level.

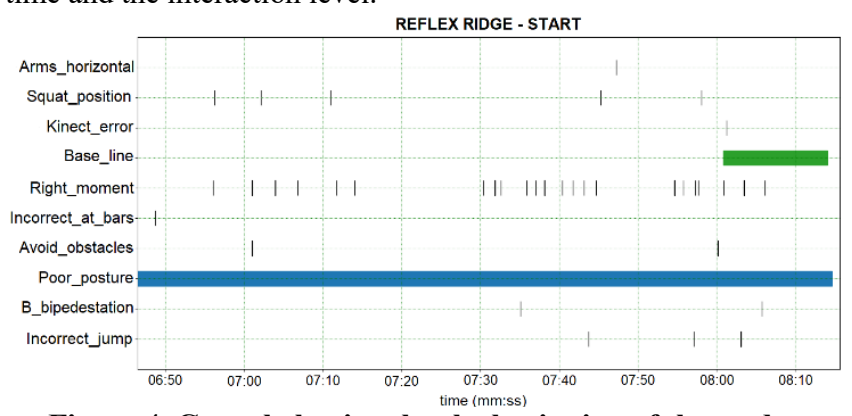

Figure 4. Game behaviors by the beginning of the study.

\subsection{Beginning of Intervention}

The initial game session was configured using the lowest game level. At this level, the interaction's difficulty is low and with few obstacles appearing on the screen, which let the player have enough time to avoid them. The collection of coins does not demand great effort from the player and the child just needs to extend her arms to do that.

The Figure 4 shows the behaviors the child exposes during her interaction with one of the exergames, at the beginning of the study. There are events associated to body posture (Poor_posture and 
Squat_position labels on Y-axis), to focalized attention and speed response (Right_moment, Incorrect_at_bars, B_bipedestation, Incorrect_jump, and Avoid obstacles), temporospatial skills (Kinect_error and Base_line), and body dissociation (Arms_horizontal). These events altogether define the psychomotor profile of the child, and the player's performance.

Dissociation. The event for the Arms_horizontal label indicates that the child failed to develop coordinated motor actions with the correct muscle dissociation so that she can extend horizontally her arms, as demanded by the gameplay.

Attention and speed response. First, the Right_moment label shows that the child reacts either late or early to the game stimulus, for instance, when she needs to jump to avoid obstacles at ankle level. This lack of attention and speed of response is observable when the child does an incorrect jump, or when she does not restore bipedestation after a squat position, which causes that obstacles hit the child.

Temporospatial. From Figure 3 one can spot how the play area was delimited with sticky tape. The base line helps as the child's reference, and to have the better detection of the child's body gestures by the Kinect sensor. The Base_line label indicates that the child failed attending the instruction to stand on or get back to this reference in order to reduce issues with the sensor device. When the child does not attend this instruction, in addition, challenges with the attention and speed of response may raise up.

Posture. Bad postures are considered related to muscle tone and label Poor_posture shows that the child keeps a bad posture along the whole game session. This bad posture is also observed with the Squat position label where events indicate the child uses such a posture for a squat position that she is kicked by obstacles.

\subsection{Middle of Intervention}

By the middle of the study, the player was playing the game at level 4 which has a major number of obstacles and with a higher rate of appearances. For the collection of coins, the child must perform various body postures, which increases its difficulty. Together with the length and speed rate of the gameplay, the player has a more demanding exercising experience.

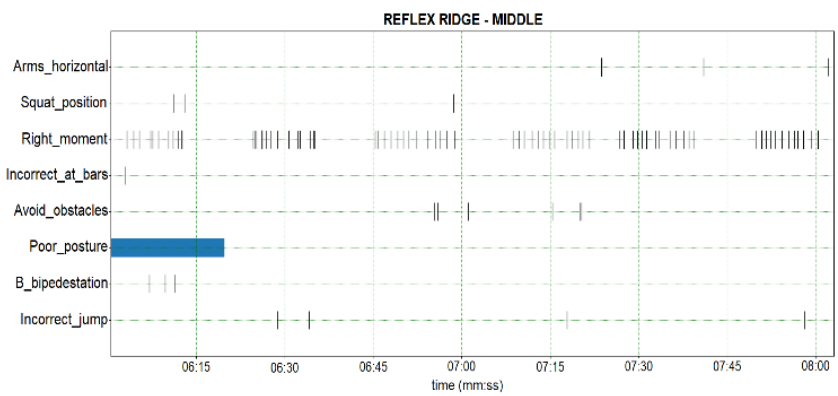

Figure 5. Game behaviors by the middle of the study.

The Figure 5 shows the game behaviors at the middle of the study. From there one can easily spot that the child adopted a better body posture and that there is a higher rate of events for the Right_moment label. Let us put this in the context of psychomotor components.

Dissociation. At this game, the child failed to organize her arms horizontally four times (halfway the $07: 15-07: 30$ period exists a couple of tagged events). We assume that these dissociation problems are associated to the speed rate of interaction with the game, which is more demanding at this level.

Attention and speed of response. It seems that the child struggles to cope with the number of events she needs to react to in time and form, as indicated by the events for the Right_moment label. Any time the child fails to react properly to the gameplay's events it leads to problems to avoid obstacles. In this game obstacles hit the child more frequently due to out-of-time jumps, late movements for bipedestation, or her squat position.

Posture. Surprisingly, at this game the child shows a bad posture only for the initial seconds of the game. This behavior could inform that the player adopted a more relaxed experience and may suggest she had a better muscle tone.

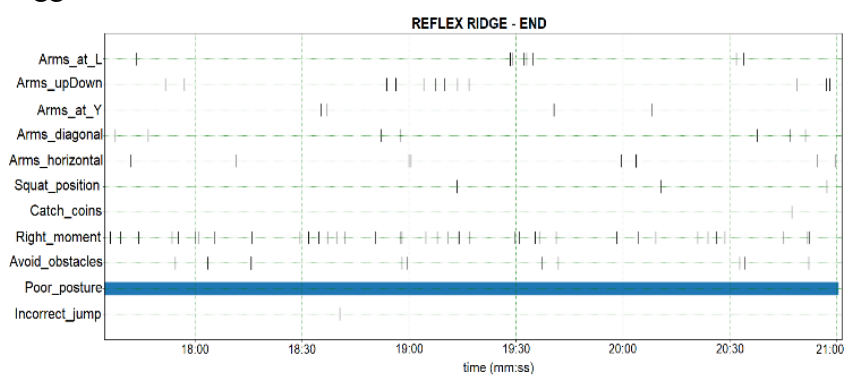

Figure 6. Game behaviors by the end of the study.

\subsection{End of Intervention}

By the end of the study the child was playing the highest game's levels, where she must structure different body's positions to collect the coins.

Dissociation. Figure 6 shows the level of dissociation problems the child faced to organize her arms hence to collect coins. Here challenges increase because the child not only needs to collect coins but also to be alert of obstacles and to the speed these show up along the gameplay.

Attention and speed response. Here we can observe a considerable number of events (Right_moment label) indicating an early or late child response to the game events. Limitations of both attention and response skills causes problems to avoid obstacles and to be hit by them.

Posture. In this game a poor body posture is observed, and again it suggests the presence of a rigid muscle tone.

Table 2. CUMANES test results

\begin{tabular}{|c|c|c|c|c|}
\hline Test & Pre & Profile & Post & Profile \\
\hline \multicolumn{5}{|c|}{ Language } \\
\hline Auditory-verbal understanding & 4 & Mid-low & 5 & Medium \\
\hline Phonological fluency & 8 & High & 7 & Mid-high \\
\hline Semantic fluency & 4 & Mid-low & 6 & Medium \\
\hline Reading comprehension & 4 & Mid-low & 4 & Mid-low \\
\hline Reading speed & 1 & Lowest & 6 & Medium \\
\hline Auditory-gnosic writing & 1 & Lowest & 1 & Lowest \\
\hline Visual perception & 2 & Low & 4 & Mid-low \\
\hline \multicolumn{5}{|c|}{ Executive function } \\
\hline Time & 2 & Low & 2 & Low \\
\hline Errors & 1 & Lowest & 4 & Mid-low \\
\hline \multicolumn{5}{|c|}{ Memory } \\
\hline Verbal memory & 3 & Low & 3 & Low \\
\hline Visual memory & 3 & Low & 1 & Lowest \\
\hline
\end{tabular}

\subsection{PRE and POST Results}

As indicated previously the CUMANES and MABC-2 tests were applied before and after the intervention phase. Remember that CUMANES evaluates global neuropsychological development which can inform about problems with learning processes and behavior. MABC-2 helps with the evaluation of motor function 
impairments by means of assessing manual dexterity and static and dynamic balance.

Table 2 shows pre and post scores the child obtained for the CUMANES test. Observe there are little improvements regarding language skills, being visual perception and reading speed the ones with major changes. For the executive function component, despite the child uses a similar time to complete the activities she makes less mistakes. This suggests an improvement with her abilities to reason about and filter interferences to complete tasks.

Table 3. MABC-2 test results
\begin{tabular}{|c|c|c|c|c|}
\hline Test & PCTL-Pre & Profile & PCTL-Post & Profile \\
\hline Manual dexterity & 3 & Difficulty & 8 & At risk \\
\hline Aim and catch & 12 & At risk & 18 & Typical \\
\hline Balance & 18 & Typical & 10 & At risk \\
\hline
\end{tabular}

Table 3 shows pre and post scores the child obtained for the MABC-2 test. Even though there is an improvement with her hand fine motor skills the percentile cut-off (PCTL) still indicates the child presents some motor difficulties. The child found problems to succeed with the balance test where activities included walk backwards on a line or hold balance on one foot for a few minutes. Nevertheless, the girl shows great performance for hand-eye coordination with the aim and catch test.

\section{Discussion}

This work aims to explore to what extent can off-the-shelf exergames stimulate psychomotor factors of children with ADHD. Gamming sessions were video recorded and video analysis was used to observe the child interaction behaviors. From the player interaction with the exergame it was observed that the game logic invites the child to use different parts of her body to interact and response to the game events. Game interaction behaviors shows that the child struggles organizing movements with her arms to collect coins. Limitations with attention and speed reaction skills are present. The child also shows problems to assess time and space, and was responding late or early to the game event, which quite often leads to be hit by obstacles. This happened for jumps, when adopting squat positions, or when she reacts late to recover bipedestation. Temporospatial limitations skills were observed from the way she used the physical game space. The child often moved forward to the wall the game was displayed on but forgotten to get back to the baseline, which sometimes causes that the Kinect device did not detect her movements. This lack of awareness for the game space we believe contributed to her low reaction speed to, for instance, avoid obstacles. Posture is another psychomotor factor the child exhibits during the gaming sessions. The child has a stooped posture most of the time.

The observations done in our study seem to be in line with theoretical works that have studied alterations in psychomotor skills in children with ADHD. For instance, the Heredad et al. work [14] reports that children with this disorder exhibit problems with three levels of motor skills: neuro-motor, motor-base and motorperceptive. Neuro-motor skills are linked to the nervous system and supported by muscle tone and dissociation, and inhibition of synkinesis and paratonias. Motor-base skills are formed by balance and dynamic coordination. Motor-perceptive skills are formed by the body schema and the spatial and temporal organization. Neuromotor skills are interesting because these are used by humans to create task-oriented movements. Some of these psychomotor alterations appear to be present in our current results.

Nevertheless, there are some psychomotor positive changes, which can also be supported with the results of the standardized tests. Despite little, there were improvements with reading speed, image comprehension and semantic fluency (CUMANES), whereas for motor skills the improvements were observable for aiming and catching (MABC).

\section{Conclusions}

The stimulation of psychomotor factors for children with learning disabilities, LD, is of great importance for different disciplines such as psychology, neurology, and academic and physical education. It takes more relevance when considering inclusive educational spaces because children with LD need to work and socialize with neurotypical peers.

In this work, we studied the contribution of off-the-shelf exergames to the stimulation of psychomotor factors for children with ADHD. In particular, we use the case of study for an elevenyear-old child, enrolled at year five on a primary school, who receives support to develop academic competences. The child was invited to play exergames and by means of it stimulate psychomotor factors and evaluate the extent to which this may reinforce superior mental functions and motor skills competences. Our preliminary findings indicate that, despite the short period of the study, the child had a marginal improvement with literacy and visuomotor coordination, but there are still challenges with equilibrium and spatiotemporal awareness.

Nevertheless, current results help us understand some level of association between psychomotor factors that a child with ADHD would face and the opportunity for using exergames as a means to reinforce cognitive, motor and affective skills, in order to help these children improve academic performance.

\section{Acknowledgments}

We thank all our participants, and to the School of the Science for the Physical Culture for providing its facilities, as well as the time and effort of all the therapists. Thanks also to the CONACYT, for supporting the project.

\section{References}

[1] Altanis, G., Boloudakis, M., Retalis, S., and Nikou, N. (2013). Children with motor impairments play a kinect learning game: first findings from a pilot case in an authentic classroom environment. J Interact Design Architect, 19, 91104.

[2] Best, J. R. (2012). Exergaming immediately enhances children's executive function. Developmental psychology, 48(5), 1501.

[3] Bogost, I. (2005). The rhetoric of exergaming. Proceedings of the Digital Arts and Cultures (DAC).

[4] Borst, C., Wieling, W., Van Brederode, J. F., Hond, A., De Rijk, L. G., and Dunning, A. J. (1982). Mechanisms of initial heart rate response to postural change. American Journal of Physiology-Heart and Circulatory Physiology, 243(5), H676H681.

[5] Bossavit, B., and Pina, A. (2014, October). Designing educational tools, based on body interaction, for children with special needs who present different motor skills. In 2014 International Conference on Interactive Technologies and Games (pp. 63-70). IEEE.

[6] Caro, K., Tentori, M., Martínez-García, A.I. and ZavalaIbarra, I. 2015. FroggyBobby: an to support children with motor problems practicing gross motor skills during 
therapeutic interventionsexergame . Journal of Computers in Human Behavior. (2015), In press.

[7] Carrasco, M. (2017). Maestría Actividad Física para la salud. Miguel Carrasco Gómez, 1-96.

[8] Castañer, M., Camerino, O., Landry, P., and Pares, N. (2016). Quality of physical activity of children in exergames: Sequential body movement analysis and its implications for interaction design. International Journal of Human-Computer Studies, 96, 67-78.

[9] Donnelly, J. E., and Lambourne, K. (2011). Classroom-based physical activity, cognition, and academic achievement. Preventive Medicine, 52(SUPPL.), S36-S42. https://doi.org/10.1016/j.ypmed.2011.01.021.

[10] Ennis, C. D. (2013). Implications of exergaming for the physical education curriculum in the 21 st century. Journal of Sport and Health Science, 2(3), 152-157. https://doi.org/10.1016/j.jshs.2013.02.004.

[11] Finco, M. D., and Maass, R. W. (2014, May). The history of exergames: promotion of exercise and active living through body interaction. In 2014 IEEE 3nd International Conference on Serious Games and Applications for Health (SeGAH) (pp. 1-6). IEEE.

[12] Gao, Z., and Chen, S. (2014). Are field-based exergames useful in preventing childhood obesity? A systematic review. Obesity Reviews, 15(8), 676-691

[13] Garris, R., Ahlers, R., and Driskell, J. E. (2002). Games, motivation, and learning: A research and practice model. Simulation \& gaming, 33(4), 441-467.

[14] Herguedas Esteban, M.C., Rubia Avi, M. and Irurtia Muñiz, M.J. (2018). Bases teóricas para la elaboración de un programa educativo de intervención psicomotriz en niños con TDAH. Revista de Educación Inclusiva, 11(1), 277-293.

[15] Hwang, S., Schneider, A. L. J., Clarke, D., Macintosh, A., Switzer, L., Fehlings, D., and Graham, T. C. (2017, June). How game balancing affects play: Player adaptation in an exergame for children with cerebral palsy. In Proceedings of the 2017 Conference on Designing Interactive Systems (pp. 699-710). ACM.

[16] Karime, A., Al Osman, H., Gueaieb, W., Alja'am, J. M., and El Saddik, A. (2011, July). Learn-pads: A mathematical exergaming system for children's physical and mental wellbeing. In 2011 IEEE International Conference on Multimedia and Expo (pp. 1-6). IEEE.
[17] Latash, M. L., and Zatsiorsky, V. (2015). Biomechanics and motor control: defining central concepts. Academic Press.

[18] Lopes, L., Santos, R., Pereira, B., and Lopes, V. P. (2013). Associations between gross Motor Coordination and Academic Achievement in elementary school children. Human Movement Science, 32(1), 9-20. https://doi.org/10.1016/j.humov.2012.05.005

[19] Pehoiu, C. (2010). Spatio-temporal orientation development during the physical education class, with 5th and 6th form pupils. World Academy of Science Engineering and Technology, 72, 76-85.

[20] Nikolić, S. J., and Ilić-Stošović, D. D. (2009). Detection and prevalence of motor skill disorders. Research in Developmental Disabilities, 30(6), 1281-1287. https://doi.org/10.1016/j.ridd.2009.05.003

[21] Sadeghi, H., Amri, S. B., Razeghi, M., Hamid, T. A., and Abdollah, M. N. H. (2017). Effects of Combined exergame and conventional exercise to reduce and prevent fall risk among elderly people: A Hypothesis. International Journal of Applied Exercise Physiology, 6(3), 80-84.

[22] Sheehan, D. P., and Katz, L. (2013). The effects of a daily, 6week exergaming curriculum on balance in fourth grade children. Journal of Sport and Health Science, 2(3), 131-137.

[23] Sun, H. (2012). Exergaming impact on physical activity and interest in elementary school children. Research quarterly for exercise and sport, 83(2), 212-220.

[24] Tomporowski, P. D., McCullick, B., Pendleton, D. M., and Pesce, C. (2015). Exercise and children's cognition: the role of exercise characteristics and a place for metacognition. Journal of Sport and Health Science, 4(1), 47-55.

[25] Westendorp, M., Hartman, E., Houwen, S., Smith, J., and Visscher, C. (2011). The relationship between gross motor skills and academic achievement in children with learning disabilities. Research in Developmental Disabilities,32(6), 2773-2779. https://doi.org/10.1016/j.ridd.2011.05.032.

[26] Wright, C. M., Duquesnay, P. J., Anzman-Frasca, S., Chomitz, V. R., Chui, K., Economos, C. D., and Sacheck, J. M. (2016). Study protocol: the Fueling Learning through Exercise (FLEX) study - a randomized controlled trial of the impact of school-based physical activity programs on children's physical activity, cognitive function, and academic achievement. BMC Public Health. https://doi.org/10.1186/s12889-016-3719-0.

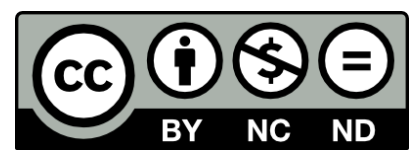

C 2020 by the authors. This work is licensed under the Creative Commons AttributionNonCommercial-NoDerivatives 4.0 International License. To view a copy of this license, visit http://creativecommons.org/licenses/by-nc-nd/4.0/ or send a letter to Creative Commons, PO Box 1866, Mountain View, CA 94042, USA. 